\title{
Does dysfunction of the mirror neuron system contribute to symptoms in Amyotrophic Lateral Sclerosis?
}

\author{
By
}

\author{
ANDREW EISEN, MD, FRCPC ${ }^{1}$ ROGER LEMON, PhD, FMedSci² MATTHEW $\underline{\text { C. KIERNAN }}$ \\ PhD,DSc,FRACP ${ }^{3}$ MICHAEL HORNBERGER PhD ${ }^{4}$ and MARTIN R. TURNER, MBBS, PhD, FRCP5
}

1. Division of Neurology, University of British Columbia, 2826 Highbury Street, Vancouver, BC., V6R 3T6, Canada.

2. Sobell Department of Motor Neuroscience and Movement Disorders, Institute of Neurology, Queen Square, London, United Kingdom.

3. Bushell Chair of Neurology, University of Sydney, Australia.

4. Department of Clinical Neurosciences, University of Cambridge, Cambridge, UK.

5. Medical Research Council and Motor Neurone Disease Association Lady Edith Wolfson Senior Clinical Fellow, Oxford University Nuffield Department of Clinical Neurosciences, Oxford, United Kingdom.

Keywords: amyotrophic lateral sclerosis; mirror neurons; empathy; motor cortex; cognition.

Word count Abstract: 129

Word count article: 3866

References: 87

Running title: Mirror Neurons in ALS.

Correspondence: Andrew Eisen MD, 2862 Highbury Street, Vancouver, BC, V6R 3T6.

Email: Eisen@mail.ubc.ca 
Declaration of interest: The authors report no conflicts of interest. The authors alone are responsible for the content and writing of the paper. 


\section{Abbreviations:}

ALS: Amyotrophic lateral sclerosis; ADM: adductor digiti minimi; APB: abductor pollicic brevis; bvFTD: Behavioral variant of frontotemporal dementia; FTD: Frontotemporal dementia; fMRI: functional MRI; MNS: Mirror neuron system; M1: Primary motor cortex; PTNs: Pyramidal tract neurons; vMPFC: Ventral medial prefrontal cortex. 


\section{Abstract}

There is growing evidence that mirror neurons, initially discovered over two decades ago in the monkey, are present in the human brain. In the monkey, mirror neurons characteristically fire not only when it is performing an action, such as grasping an object, but also when observing a similar action performed by another agent (human or monkey). In this review we discuss the origin, cortical distribution and possible functions of mirror neurons as a background to exploring their potential relevance in amyotrophic lateral sclerosis (ALS). We have recently proposed that ALS (and the related condition of frontotemporal dementia) may be viewed as a failure of interlinked functional complexes having their origins in key evolutionary adaptations. The mirror neuron system is considered by many to be the basis of primates' social cognition, with a clear evolutionary advantage. Impaired empathy and motor control has been related to a defective mirror neuron system. In ALS, the mirror neuron system might be implicated in empathy, the split-hand syndrome, gait, speech, and related languagegesture impairments. 


\section{Introduction}

The human motor system has been critically refined through evolution and is profoundly interconnected to the many other brain networks. Motor system degeneration, involving loss of limb function, gait, speech and vocalization are the clinical hallmarks of the adult neurodegenerative disorder amyotrophic lateral sclerosis (ALS), and can be understood, to an extent, by this evolutionary development (Eisen et al. , 2014b). Although, typically confined to a body region at disease onset, there may be a rapid coalescence of regional involvement involving upper and lower motor neuron loss, resulting in a characteristic clinical syndrome, termed ALS (Hardiman et al. , 2011, Kiernan et al. , 2011). Respiratory failure is the terminal event in about $85 \%$ of patients with ALS, a characteristic feature of ALS that is absent in most other Neurodegenerationsneurodegenerations. ALS also affects extramotor systems, involving clinical, pathological and genetic overlap with frontotemporal dementia (FTD), most commonly through expansions of the hexanucleotide repeat in C9orf72 (DeJesusHernandez et al. , 2011, Renton et al. , 2011). Members of the same family may develop relatively 'pure' FTD, ALS or both, thus broadening the clinical syndrome of ALS (Snowden et al. , 2013). The incidence of frank dementia in sporadic ALS is approximately 15\%, but a much higher proportion of patients show sub-clinical evidence of executive dysfunction (Lillo et al. , 2011). Executive functions, free verbal recall and naming are the most affected cognitive deficits in ALS-FTD variants (Strong, 2008, Rascovsky et al. , 2011, Snowden et al. , 2013). However, it is also appreciated that effective social interactions in everyday life (social cognition), and its consequences (social cognitive deficits) are increasingly apparent in neurodegenerative disorders, including ALS (Elamin et al. , 2012).

While most clinical research in ALS has understandably focused on motor pathways, attention is now being directed to all facets of the motor pathways (reflecting ALS as a motor system disorder). Of relevance, mirror neurons and the mirror neuron system (MNS), are special in being active both 5 Eisen et al, Mirror Neurons in ALS 
during motor action and during observation of another individual's motor action, so that the same neuron has motor and sensory representations (Rizzolatti and Craighero, 2004; Keysers and Gazzola, 2006). When observing an action two distinct types of information are obtained - what action is being done and, more complex - what for or why? The 'matching hypothesis' suggests that whenever individuals observe an action being done by someone else, mirror neurons that code for that action are activated in the observer. Since the observers are aware of the outcome of their own motor acts, they also recognize what the other individual is doing without the need for further cognitive processing (Rizzolatti et al. , 2009).

While mirror neurons were discovered over 20 years ago, questions remain regarding their developmental origins and function. Much has been written about a possible role in neurological disorders, but our current knowledge of mirror neurons and the input and output systems to which they belong is still rudimentary, so that it is difficult to predict with any certainty the likely consequences of mirror neuron dysfunction. Nevertheless, given that mirror neurons could be of fundamental importance in social cognition, as well as functioning in the processes of motor control and motor learning (Rizzolatti, 2008), it seems worthwhile considering the possible role of mirror neurons in the pathophysiology of ALS. Here we discuss some basic aspects of the MNS and explore how, when disordered, mirror neurons may have relevance in early symptomatology of ALS. In particular we make the case for possible links between mirror neuron function and empathy, hand function, gait and articulation, all of which are significantly impaired in ALS.

\section{Mirror Neuron Development and Function}

From an evolutionary point of view, it seems reasonable that there may be some innate mechanisms in place that would be facilitated through sensorimotor learning (Del Giudice et al. , 2009). Indeed the 
MNS might inform the Sapient Paradox (Renfrew, 2008), broadly encapsulated as the 'hardware' for the human neocortex preceding any evidence for higher cognitive outputs by tens of thousands of years. The settling of humans into communities may have been a key factor, in effect the brain being only as productive as those it is shared with, a process in which mirror neurons would be expected to play a crucial role.

While there is no firm evidence for the existence of a mirror neurons at birth, there is general agreement that infants at birth are attracted to specific sets of stimuli, including faces, their own hands, and especially their own hands in motion (Casile et al. , 2011, Ferrari et al. , 2013). This may provide sensorimotor experiences that are the necessary scaffolding for mirror neuron development. Empathy, in which mirror neurons have also been implicated (see below), is observable even during the first day of life (Singer, 2006), and infant data using eye-tracking measures suggest that the MNS develops before 12 months of age (Corradini et al. , 2013). In further support, EEG-mu rhythm desynchronization has been widely used as an indicator of a functioning MNS. However, this is of course an indirect measure, and cannot prove the existence of populations of mirror neurons that are activated during both action execution and observation, as opposed to separate populations of 'sensory' and 'motor'-related neurons (Braadbaart et al. , 2013). That said, it is of interest that this type of desynchronization has also been shown in infants during action observation (Virji-Babul et al. , 2012).

The roles proposed for a MNS include understanding the meaning of behaviors; helping to learn motor tasks or to choose our own actions (Bonini et al. , 2011, Caggiano et al. , 2012); and predicting the actions we are observing, without assigning a goal to them (Bonini et al. , 2011, Marshall, 2014). It has been suggested that frontoparietal areas provide the basis for bridging the gap between the physical 7 Eisen et al, Mirror Neurons in ALS 
self and others through motor-simulation mechanisms and cortical midline structures engage in processing information about the self and others in more abstract, evaluative terms (Uddin et al. , 2007). However, there may exist many classes of mirror neurons, influenced by different aspects of an observed action (e.g., type of the observed motor act, distance of the observer from the observed action, point of view from which the action is observed)(Casile et al. , 2011).

\section{Cerebral Localisation}

In non-human primates, activity of mirror neurons have been found in different regions of the motor system (Lemon, 2010), and mirror neurons have been identified in the ventral premotor cortex (see review by Kilner and Lemon, 2013), primary motor cortex (Vigneswaran et al. , 2013), inferior parietal lobule (Casile, 2013), and ventral and lateral intraparietal areas. In contrast the existence of a MNS in humans, has been surmised almost entirely by functional MRI (fMRI) and Mu rhythm in the EEG a measure of a resting motor state, which is normally suppressed by input from an action observation or movement execution. This characteristic has caused Mu suppression to be used as proxy marker for mirror neuron activation (Braadbaart et al. , 2013). However, mu suppression involves a range of structures that modulate motor preparation and are sensitive to visual input, including but not restricted to the human analogue of the mirror neuron system (Braadbaart et al. , 2013).

Many fMRI studies have shown a broad overlap between frontal cortical areas activated for both action execution and observation, although, just as with the mu-desynchronization, this is not evidence for the existence of mirror neurons in the human brain (see Cerri et al. 2014). Areas of overlap do include the ventral premotor cortex, although comparison of fMRI studies in humans versus macaques have demonstrated that fMRI activation of the specific subdivision of PMv that is known to contain mirror neurons (F5c) requires that the actor is in full view, and not just the actor's hand (Nelissen et al., 2005; 2011).

8 Eisen et al, Mirror Neurons in ALS 


\section{ALS Hand Function and Mirror Neurons}

Awkwardness in tasks requiring fine finger movements (e.g., difficulties with buttons), stiffness of the fingers, and weakness or wasting of the hand muscles may be early symptoms in ALS, though not specific to this disorder. Pincer and precision grip are particularly vulnerable in ALS however, which is uniquely manifested as a split hand syndrome, where there is preferential involvement of the lateral, thenar-innervated muscles (abductor pollicis brevis and first dorsal interosseous) as compared to the hypothenar-innervated muscles (abductor digiti minimi) (Eisen et al. , 2012, Menon et al. , 2013). Evidence using transcranial magnetic stimulation suggests that the split hand of ALS is cortical in origin, and in particular due to failure of the thenar hand corticomotneurons arising in M1 cortex (Weber et al. , 2000). Also, cortical hyperexcitability, a consistent feature of ALS (Vucic et al. , 2006), has been shown to be associated with the split hand in ALS (Bae et al. , 2014). Peripherally, motor axons innervating abductor pollicis brevis (APB) have physiologically higher excitability than those supplying abductor digiti minimi (ADM), and peripheral hyperexcitabilty in ALS in more pronounced in APB than ADM axons (Shibuya et al. , 2013).

Accurate pincer grip is dependent on force adjustments of the fingers and hand, occurring in anticipation of the relevant action required (Johansson et al. , 1988, Serrien et al. , 1999, Venkadesan, 2008). This involves a feed-forward regulation requiring the use of sensorimotor memory, as grip force parameters, such as object weight and friction between skin and object surface, are recalled from previous experience (Johansson et al. , 1988, Danion et al. , 2007).

The primary motor cortex plays a crucial role in the regulation of grip force during object manipulation, influencing distal arm, hand, and finger muscles engaged in grasping, especially, but not exclusively, through monosynaptic cortico-motoneuronal connections with motoneurons in the spinal 9 Eisen et al, Mirror Neurons in ALS 
cord (Lemon et al. , 1986, Maier et al. , 1993). Current evidence indicates that human hand-related mirror neurons are sensitive to object- and goal-directed behavior, not just biological motion (Enticott et al. , 2010). However, the main activity of the MNS during the execution and the observation of precision grasping pantomime may be located in the inferior parietal lobe rather than premotor areas (Plata Bello et al. , 2014). In ALS grip force is impaired and has been shown that there is greater force used than needed, when compared to controls (Nowak et al. , 2003).

The role of mirror neurons in primary motor cortex presents a puzzle. In the macaque monkey, a significant proportion of pyramidal tract neurons (PTNs) in the primary motor cortex hand area exhibit mirror-like activity (Kraskov et al. , 2014). Given that these neurons have traditionally been associated with active execution of motor tasks, it may at first seem surprising that many show significant modulation of their discharge during action observation. Even more surprising that a few examples were reported of PTNs which were also identified as having cortico-motoneuronal postspike effects on intrinsic hand muscles (Kraskov et al. , 2014). However, a large fraction of the mirror PTNS in M1 showed suppression of discharge during action observation (Kraskov et al. , 2014); some cortico-motoneuronal PTNs also showed this suppression. One suggested role for these suppression mirror neurons relates to involvement in withholding unwanted movement during action observation, and that such a braking mechanism would avoid unintended overflow of activity (Schieber, 2011). Such overflow is also prevented because, compared with execution, M1 PTNs show greatly attenuated activity during action observation. It remains to be confirmed that suppression mirror neurons are present in humans (Mukamel et al., 2010), but there are daily occasions when one wants to covertly imitate an observed action, allowing the observer to watch the actor, while using their own motor system to identify and categorize the observed movement, without the activation overflowing into selfmovement (Vigneswaran et al. , 2013). 


\section{Gait and Walking}

In both young and older subjects, a network comprising the supplementary motor cortex, primary motor cortex, right prefrontal cortex, and cerebellum, (regions in which MNs are active) is activated during motor imagery of gait (Allali et al. , 2014). But these imaging recordings have not been replicated by cellular recording in the prefrontal cortex or cerebellum. There is an age-related increase in brain activity in the supplementary motor area, orbitofrontal cortex, and dorsolateral frontal cortex. Hippocampal activity is modulated by task difficulty in the elderly participants.(Allali et al. , 2014).

When subjects watched a video clip showing an actor standing and walking in an egocentric perspective, compared to observing a clip of the clutch movement of a right hand, activation occurred in supplementary motor area, bilateral precentral gyrus, left dorsal premotor cortex, and cingulate motor area (Wang et al. , 2008). The tasks used in this study were considered to engage both mirror neurons and mental imagery. Action observation training and motor imagery, both presumed to involve mirror neuron activation, were shown to improve balance and gait, significantly better than conventional treatment used after a stroke (Kim et al. , 2013).

\section{A role in empathy}

Empathy is essential for human social interaction, allowing us to share and respond to the emotional experience of others in reference to ourselves (Decety, 2011, Decety et al. , 2012). Empathy involves experiencing emotion vicariously, and understanding the reasons for those emotions. Multiple brain regions are involved in empathy processing with anterior cingulate and anterior insular regions being of particular importance (Saxe, 2006, Singer, 2006). Nevertheless, there has been also the suggestion 
that the MNS is involved in empathy processing by providing an action observation framework for empathy with others. This may suggest that empathy is hard-wired in the brain as action observation automatically triggers empathetic processes. Indeed, it has been shown that people who are more empathic have stronger activations of the putative mirror system for hand actions and also the mirror system for emotions, supporting the notion that the MNS is linked to empathy (Acharya et al. , 2012, Zaki et al. , 2012).

Still, empirical support linking empathy and MNS directly is still lacking and there is increasingly the suggestion that the MNS does not have an 'interpretative' role in empathy processing (i.e. emotionally interpreting the actions observed), instead it provides a top-down generated, predictive simulation of action understanding (Kable et al. , 2005, Csibra, 2008). This does not mean that the MNS is not involved in empathy processing, instead it provides an action observation aspect that might facilitate the emotional resonance, with observing someone else being happy/sad/hurt increasing your emotional reaction. However, one can have empathy without any action observation, i.e. reading in a novel or newspaper about the joy or plight of others, may elicit strong empathic sentiments as well (Lamm et al. , 2011).

The notion that the MNS might act more as an 'empathetic facilitator', instead of being part of the empathetic system itself also nicely dovetails with neurodegenerative findings. In particular, findings in behavioral variant of frontotemporal dementia (bvFTD), which shows severe loss of empathy even recognized in the diagnostic criteria (Piguet et al. , 2011, Rascovsky et al. , 2011), report that loss of empathy is more attributed to atrophy in the ventral medial prefrontal cortex (vMPFC), including anterior cingulate regions and not the MNS system per se (Shamay-Tsoory et al. , 2004, Lough et al. , 2006). Interestingly, similar vMPFC atrophy in bvFTD has been attributed to emotional blunting (Berenbaum et al. , 1987, Rosen et al. , 2002) and loss of insight (Hornberger et al. , 2014), highlighting the critical function of this region in affective perspective-taking (i.e. putting yourself in someone 12 Eisen et al, Mirror Neurons in ALS 
else's shoes), which is required for empathy to occur. Thus, in bvFTD the apparent empathy deficits might be more due to the failure of taking an affective perspective instead of action observation deficits, per se. The deficits in anterior cingulate/vMPFC associated with loss of empathy in bvFTD are of particular interest for ALS, where patients show consistently changes in anterior cingulate regions as well (Lillo et al. , 2012, Filippi et al. , 2013). Indeed, recent studies suggest that impairments of emotional decision-making, and impaired empathy might be also more due to anterior cingulate and insula atrophy changes than ventral motor cortex atrophy in ALS (Girardi et al. , 2011, Cerami et al. , 2014). If so, this would suggest that empathy impairments in ALS might be also more due to perspective taking deficits than pure action observation deficits similar to bvFTD.

At a clinical level, empathy deficits in ALS are not as evident as observed in patients diagnosed with bvFTD. However, a potential relationship might exist between empathy deficits and the prevalent apathy in ALS, which is commonly detected on a clinical level. Indeed, apathy has been described has having 3 underlying mechanisms (emotional-affective; cognitive; auto-activation) (Stuss et al. , 2000, Levy et al. , 2006). In particular, emotional-affective apathy, described as an emotional blunting with reactivity to emotional situations poor and short-lived (Levy et al. , 2006), might be closely related to empathy deficits in ALS. This is nicely corroborated by many clinicians' reports of ALS patients being surprisingly passive and accepting faced with such a catastrophic diagnosis, as the emotional blunting might have reduced the emotional reactivity to the diagnosis in the patients. Further, both empathy and emotional-affective apathy have been linked to dysfunction in vMPFC and particularly the anterior cingulate (Rosen et al. , 2002) and thus a strong link between empathy and apathy might exist in ALS. Clearly this topic needs further future investigations to elucidate in more detail the roles of the MNS and apathy in the generation of empathy deficits in ALS. 
In terms of motor function more generally, both motor imagery and action observation have been shown to play a role in learning or re-learning complex motor tasks. They may share a common neurophysiological basis in the MNS (Gatti et al. , 2013). Motor imagery, the ability to imagine movements, is defective in ALS (Stanton 2007) (Fiori et al. , 2013). In ALS, motor imagery has been shown to share similar neural networks (the primary motor, premotor, and supplementary motor cortex) as motor execution, as this is not different from normal subjects (Lule et al. , 2007). In contrast, there is reduced activation for motor imagery versus motor execution, in several other cortical areas, including the left inferior parietal lobule, and in the anterior cingulate gyrus and medial pre-frontal cortex (Stanton et al. , 2007).

\section{Mirror neurons in language and gesture}

It has been proposed that gesture and speech form a single system of communication, because they are linked to the same thought processes, even if expressed differently (Arbib et al. , 2008, Cartmill et al. , 2012, Eisen et al. , 2014b). Observing and executing the grasp of small and large objects, for example a grape versus an apple, changes the degree of mouth opening and simultaneously pronunciation of syllables (Gentilucci et al. , 2008b). Frequently we pronounce words while executing gestures expressing the same meaning as the word. While pronouncing "OK", one often forms a circle with the forefinger and thumb in contact at their tips, while the rest of the fingers extend outward (Gentilucci et al. , 2008a). Manual gestures predate the early development of speech in children and predict later success. The mirror neuron system could have provided a natural platform for the subsequent evolution of language. In non-human primates, the system provides for the understanding of action/imitation, both prerequisites for language (Corballis, 2010). However, others have argued strongly against the involvement of the mirror neuron system in language (Hickok et al. , 2014). 
Electromyography has demonstrated concurrent contraction of the orbicularis oris, when participants execute a precision grip, pointing with the index finger, or curling the fingers. Concurrent contraction of other facial muscles not directly involved in articulation was not observed during these acts (Higginbotham et al. , 2008). This observation mimics the hand-mouth activity in non-human primates and prelinguistic infants, and the influence of grasping on labial articulation in adult humans (Gentilucci et al. , 2001). It also supports the idea that there is a transition from gestural to verbal, articulate communication systems. However any involvement of mirror neurons in this transition is still unproven.

FreeThe free-flowing and intricately varied speech of humans; requires very fine control of breathing, attained by means of a major increase in thoracic innervation associated with monosynaptic connections to both inspiratory and expiratory musculature (Maclarnon, 2004). Bulbar-onset ALS most obviously impairs vocalization and swallowing but; also significantly impacts respiratory dysfunction through the evolutionarily conserved pre-Botzinger brainstem complex responsible for respiratory patterning (Cinelli et al. , 2013). A bulbar-mediated component in ALS is emotionality, with inappropriate crying and laughing. Emotional crying appears to be uniquely human (Trimble, 2012). A central coordinator of the nuclei at the mesencephalic-pontine junction innervates the muscles involved in facial expression, respiration, and phonation (Arias, 2011), and receives inhibitory connections from the premotor and motor cortex, and excitatory input from the temporal cortex, amygdala, and hypothalamus.

Early in the course of ALS, when symptoms are mild, speech typically appears normal. With disease progression, the voice quality may become strained, with a slow speaking rate involving monopitch and monoloudness. These impairments reflect failure of highly coordinated movements of the tongue, lips, and jaw, a prerequisite for normal articulation. Early in the disease speech patterns are consistent during typical speech, indicating successful adaption to disease-related articulatory deficits. However, 15 Eisen et al, Mirror Neurons in ALS 
during fast speech, patterns become inconsistent indicating impaired articulatory control when the speech motor system operates near its performance limit (Mefferd et al. , 2014). In some patients with isolated speech deficits, FDG-PET studies, have shown subtle hypometabolism affecting the premotor and motor cortices (Clark et al. , 2014).

Narrative discourse is an essential part of everyday communication. A recent study demonstrated that patients with ALS have a disorder of narrative expression that cannot be fully explained by their motor disorder (Ash et al. , 2014). This was true of patients without overt dysarthria or executive function defects. Based on limited cortical imaging (gray matter atrophy and white matter reduced fractional anisotropy) the impaired narrative discourse was considered to be related in part to executive limitations that interfere with the planning and organization needed for narrative expression and to disruption of a large-scale neural network in the frontal lobe that appears to support narrative discourse (Ash et al. , 2014). The study did not correlate the narrative defect with EMG of orofacial muscles.

\section{Conclusion}

The study of mirror neurons generates both interest and controversy. Mirror neurons respond to actions that we observe in others, and discharge in the same way when we recreate that action ourselves. But And perhaps they may well be involved in a myriad of other sophisticated aspects of human behavior and thought processes yet to be fully deciphered. Numerous $\underline{-1 n}$ support, numerous single unit recording studies have elucidated mirror neuron characteristics in different cortical regions of the non-human primate brain, and the presence of single mirror neurons has been demonstrated in humans (Mukamel et al. , 2010). In general it is probably more appropriate in humans to refer to a fronto-parietal mirror neuron system (MNS). Failure of this system has been 16 Eisen et al, Mirror Neurons in ALS 
considered important in disparate conditions including autism spectrum disorders (Hamilton, 2013), schizophrenia (Mehta et al. , 2014), Alzheimer's disease and Parkinson's disease (Alegre et al. , 2011).

Returning then to ALS, is it conceivable that involvement of a mirror neuronal system may form part of a neurodegenerative cascade? If it is accepted that impaired empathy and defective motor function with impaired motor control are all features of ALS, that may indeed suggest failure of the MNS. But realistically, current knowledge of human mirror neurons and their functional relation with other brain systems makes this a difficult hypothesis to formally assess. Nevertheless, because ALS affects corticospinal neurons and the latter show mirror-like properties, their failure has likely effects beyond those typically ascribed to upper motor neuron dysfunction in ALS.

A significant difficulty in identifying impairment of the MNS in ALS, and other neurodegenerative diseases, is the slowly progressive nature of these disorders, and their prolonged preclinical period (Eisen et al. , 2014a, Thomsen et al. , 2014). This presumably enables a degree of adaption, masking subtle abnormalities in the MNS, which might otherwise induce clinical deficits. As-however, it remains plausible that as imaging and electrophysiological recording become more refined, the human MNS is likely to reveal important pathophysiological data related to ALS, which in turn is hoped will aid in early diagnosis and thereby better intervention. 


\section{References:}

Acharya S, Shukla S. Mirror neurons: Enigma of the metaphysical modular brain. J Nat Sci Biol Med. 2012;3:118-24.

Alegre M, Guridi J, Artieda J. The mirror system, theory of mind and Parkinson's disease. J Neurol Sci. 2011;310:194-6.

Allali G, van der Meulen M, Beauchet 0, Rieger SW, Vuilleumier P, Assal F. The neural basis of agerelated changes in motor imagery of gait: an FMRI study. J Gerontol A Biol Sci Med Sci. 2014;69:138998.

Arbib MA, Liebal K, Pika S. Primate vocalization, gesture, and the evolution of human language. Curr Anthropol. 2008;49:1053-63; discussion 63-76.

Arias M. [Neurology of laughter and humour: pathological laughing and crying]. Rev Neurol. 2011;53:415-21.

Ash S, Menaged A, Olm C, McMillan CT, Boller A, Irwin DJ, et al. Narrative discourse deficits in amyotrophic lateral sclerosis. Neurology. 2014;83:520-8.

Bae JS, Menon P, Mioshi E, Kiernan MC, Vucic S. Cortical hyperexcitability and the split-hand plus phenomenon: pathophysiological insights in ALS. Amyotroph Lateral Scler Frontotemporal Degener. 2014;15:250-6.

Berenbaum SA, Abrams R, Rosenberg S, Taylor MA. The nature of emotional blunting: a factor-analytic study. Psychiatry Res. 1987;20:57-67.

Bonini L, Ferrari PF. Evolution of mirror systems: a simple mechanism for complex cognitive functions. Ann N Y Acad Sci. 2011;1225:166-75.

Braadbaart L, Williams JH, Waiter GD. Do mirror neuron areas mediate mu rhythm suppression during imitation and action observation? Int J Psychophysiol. 2013;89:99-105.

Caggiano V, Fogassi L, Rizzolatti G, Casile A, Giese MA, Thier P. Mirror neurons encode the subjective value of an observed action. Proc Natl Acad Sci U S A. 2012;109:11848-53.

Cartmill EA, Beilock S, Goldin-Meadow S. A word in the hand: action, gesture and mental representation in humans and non-human primates. Philos Trans R Soc Lond B Biol Sci.

2012;367:129-43.

Casile A. Mirror neurons (and beyond) in the macaque brain: An overview of 20 years of research. Neurosci Lett. 2013;540:3-14.

Casile A, Caggiano V, Ferrari PF. The mirror neuron system: a fresh view. Neuroscientist. 2011;17:52438.

Cerami C, Dodich A, Canessa N, Crespi C, Iannaccone S, Corbo M, et al. Emotional empathy in amyotrophic lateral sclerosis: a behavioural and voxel-based morphometry study. Amyotroph Lateral Scler Frontotemporal Degener. 2014;15:21-9.

Cinelli E, Robertson B, Mutolo D, Grillner S, Pantaleo T, Bongianni F. Neuronal mechanisms of respiratory pattern generation are evolutionary conserved. J Neurosci. 2013;33:9104-12. Clark HM, Duffy JR, Whitwell JL, Ahlskog JE, Sorenson EJ, Josephs KA. Clinical and imaging characterization of progressive spastic dysarthria. Eur J Neurol. 2014;21:368-76.

Corballis MC. Mirror neurons and the evolution of language. Brain Lang. 2010;112:25-35.

Corradini A, Antonietti A. Mirror neurons and their function in cognitively understood empathy. Conscious Cogn. 2013;22:1152-61.

Csibra G. Goal attribution to inanimate agents by 6.5-month-old infants. Cognition. 2008;107:705-17. Danion F, Sarlegna FR. Can the human brain predict the consequences of arm movement corrections when transporting an object? Hints from grip force adjustments. J Neurosci. 2007;27:12839-43. 
Decety J. The neuroevolution of empathy. Ann N Y Acad Sci. 2011;1231:35-45.

Decety J, Svetlova M. Putting together phylogenetic and ontogenetic perspectives on empathy. Dev Cogn Neurosci. 2012;2:1-24.

DeJesus-Hernandez M, Mackenzie IR, Boeve BF, Boxer AL, Baker M, Rutherford NJ, et al. Expanded GGGGCC hexanucleotide repeat in noncoding region of C90RF72 causes chromosome 9p-linked FTD and ALS. Neuron. 2011;72:245-56.

Del Giudice M, Manera V, Keysers C. Programmed to learn? The ontogeny of mirror neurons. Dev Sci. 2009;12:350-63.

Eisen A, Kiernan M, Mitsumoto H, Swash M. Amyotrophic lateral sclerosis: a long preclinical period? J Neurol Neurosurg Psychiatry. 2014a;85:1232-8.

Eisen A, Kuwabara S. The split hand syndrome in amyotrophic lateral sclerosis. J Neurol Neurosurg Psychiatry. 2012;83:399-403.

Eisen A, Turner MR, Lemon R. Tools and talk: An evolutionary perspective on the functional deficits associated with amyotrophic lateral sclerosis. Muscle Nerve. 2014b;49:469-77.

Elamin M, Pender N, Hardiman 0, Abrahams S. Social cognition in neurodegenerative disorders: a systematic review. J Neurol Neurosurg Psychiatry. 2012;83:1071-9.

Enticott PG, Kennedy HA, Bradshaw JL, Rinehart NJ, Fitzgerald PB. Understanding mirror neurons: evidence for enhanced corticospinal excitability during the observation of transitive but not intransitive hand gestures. Neuropsychologia. 2010;48:2675-80.

Ferrari PF, Tramacere A, Simpson EA, Iriki A. Mirror neurons through the lens of epigenetics. Trends Cogn Sci. 2013;17:450-7.

Filippi M, Agosta F, Scola E, Canu E, Magnani G, Marcone A, et al. Functional network connectivity in the behavioral variant of frontotemporal dementia. Cortex. 2013;49:2389-401.

Fiori F, Sedda A, Ferre ER, Toraldo A, Querzola M, Pasotti F, et al. Exploring motor and visual imagery in Amyotrophic Lateral Sclerosis. Exp Brain Res. 2013;226:537-47.

Gatti R, Tettamanti A, Gough PM, Riboldi E, Marinoni L, Buccino G. Action observation versus motor imagery in learning a complex motor task: a short review of literature and a kinematics study.

Neurosci Lett. 2013;540:37-42.

Gentilucci M, Benuzzi F, Gangitano M, Grimaldi S. Grasp with hand and mouth: a kinematic study on healthy subjects. J Neurophysiol. 2001;86:1685-99.

Gentilucci M, Dalla Volta R. Spoken language and arm gestures are controlled by the same motor control system. Q J Exp Psychol (Hove). 2008a;61:944-57.

Gentilucci M, Dalla Volta R, Gianelli C. When the hands speak. J Physiol Paris. 2008b;102:21-30. Girardi A, Macpherson SE, Abrahams S. Deficits in emotional and social cognition in amyotrophic lateral sclerosis. Neuropsychology. 2011;25:53-65.

Hamilton AF. Reflecting on the mirror neuron system in autism: a systematic review of current theories. Dev Cogn Neurosci. 2013;3:91-105.

Hardiman 0, van den Berg LH, Kiernan MC. Clinical diagnosis and management of amyotrophic lateral sclerosis. Nat Rev Neurol. 2011;7:639-49.

Hickok G, Rogalsky C, Chen R, Herskovits EH, Townsley S, Hillis AE. Partially overlapping sensorimotor networks underlie speech praxis and verbal short-term memory: evidence from apraxia of speech following acute stroke. Front Hum Neurosci. 2014;8:649.

Higginbotham DR, Isaak MI, Domingue JN. The exaptation of manual dexterity for articulate speech: an electromyogram investigation. Exp Brain Res. 2008;186:603-9.

Hornberger M, Yew B, Gilardoni S, Mioshi E, Gleichgerrcht E, Manes F, et al. Ventromedial-frontopolar prefrontal cortex atrophy correlates with insight loss in frontotemporal dementia and Alzheimer's disease. Hum Brain Mapp. 2014;35:616-26. 
Johansson RS, Westling G. Programmed and triggered actions to rapid load changes during precision grip. Exp Brain Res. 1988;71:72-86.

Kable JW, Kan IP, Wilson A, Thompson-Schill SL, Chatterjee A. Conceptual representations of action in the lateral temporal cortex. J Cogn Neurosci. 2005;17:1855-70.

Kiernan MC, Vucic S, Cheah BC, Turner MR, Eisen A, Hardiman O, et al. Amyotrophic lateral sclerosis. Lancet. 2011;377:942-55.

Kim JH, Lee BH. Action observation training for functional activities after stroke: a pilot randomized controlled trial. NeuroRehabilitation. 2013;33:565-74.

Kraskov A, Philipp R, Waldert S, Vigneswaran G, Quallo MM, Lemon RN. Corticospinal mirror neurons. Philos Trans R Soc Lond B Biol Sci. 2014;369:20130174.

Lamm C, Decety J, Singer T. Meta-analytic evidence for common and distinct neural networks associated with directly experienced pain and empathy for pain. Neuroimage. 2011;54:2492-502. Lemon RN. What drives corticospinal output? F1000 Biol Rep. 2010;2:51.

Lemon RN, Mantel GW, Muir RB. Corticospinal facilitation of hand muscles during voluntary movement in the conscious monkey. J Physiol. 1986;381:497-527.

Levy R, Dubois B. Apathy and the functional anatomy of the prefrontal cortex-basal ganglia circuits. Cereb Cortex. 2006;16:916-28.

Lillo P, Mioshi E, Zoing MC, Kiernan MC, Hodges JR. How common are behavioural changes in amyotrophic lateral sclerosis? Amyotroph Lateral Scler. 2011;12:45-51.

Lillo P, Savage S, Mioshi E, Kiernan MC, Hodges JR. Amyotrophic lateral sclerosis and frontotemporal dementia: A behavioural and cognitive continuum. Amyotroph Lateral Scler. 2012;13:102-9. Lough S, Kipps CM, Treise C, Watson P, Blair JR, Hodges JR. Social reasoning, emotion and empathy in frontotemporal dementia. Neuropsychologia. 2006;44:950-8.

Lule D, Diekmann V, Kassubek J, Kurt A, Birbaumer N, Ludolph AC, et al. Cortical plasticity in amyotrophic lateral sclerosis: motor imagery and function. Neurorehabil Neural Repair. 2007;21:51826.

Maclarnon A, Hewitt, G. Increased Breathing Control: Another Factor in the Evolution of Human Language. Evol Anthropol. 2004;13:181-97.

Maier MA, Bennett KM, Hepp-Reymond MC, Lemon RN. Contribution of the monkey corticomotoneuronal system to the control of force in precision grip. J Neurophysiol. 1993;69:772-85. Marshall J. Mirror neurons. Proc Natl Acad Sci U S A. 2014;111:6531.

Mefferd AS, Pattee GL, Green JR. Speaking rate effects on articulatory pattern consistency in talkers with mild ALS. Clin Linguist Phon. 2014;28:799-811.

Mehta UM, Thirthalli J, Basavaraju R, Gangadhar BN, Pascual-Leone A. Reduced mirror neuron activity in schizophrenia and its association with theory of mind deficits: evidence from a transcranial magnetic stimulation study. Schizophr Bull. 2014;40:1083-94.

Menon P, Bae JS, Mioshi E, Kiernan MC, Vucic S. Split-hand plus sign in ALS: differential involvement of the flexor pollicis longus and intrinsic hand muscles. Amyotroph Lateral Scler Frontotemporal Degener. 2013;14:315-8.

Mukamel R, Ekstrom AD, Kaplan J, Iacoboni M, Fried I. Single-neuron responses in humans during execution and observation of actions. Curr Biol. 2010;20:750-6.

Nowak DA, Hermsdorfer J, Topka H. When motor execution is selectively impaired: control of manipulative finger forces in amyotrophic lateral sclerosis. Motor Control. 2003;7:304-20.

Piguet O, Hornberger M, Mioshi E, Hodges JR. Behavioural-variant frontotemporal dementia: diagnosis, clinical staging, and management. Lancet Neurol. 2011;10:162-72.

Plata Bello J, Modrono C, Marcano F, Gonzalez-Mora JL. The mirror neuron system and motor dexterity: what happens? Neuroscience. 2014;275:285-95. 
Rascovsky K, Hodges JR, Knopman D, Mendez MF, Kramer JH, Neuhaus J, et al. Sensitivity of revised diagnostic criteria for the behavioural variant of frontotemporal dementia. Brain. 2011;134:2456-77. Renfrew C. Neuroscience, evolution and the sapient paradox: the factuality of value and of the sacred. Philos Trans R Soc Lond B Biol Sci. 2008;363:2041-7.

Renton AE, Majounie E, Waite A, Simon-Sanchez J, Rollinson S, Gibbs JR, et al. A hexanucleotide repeat expansion in C90RF72 is the cause of chromosome 9p21-linked ALS-FTD. Neuron. 2011;72:257-68. Rizzolatti G, Fabbri-Destro M, Cattaneo L. Mirror neurons and their clinical relevance. Nat Clin Pract Neurol. 2009;5:24-34.

Rizzolatti G, Sinigaglia, C. Mirrors in the Brain: How our minds share acrtions, emotions and experience. Oxford, UK: Oxford University Press; 2008.

Rosen HJ, Gorno-Tempini ML, Goldman WP, Perry RJ, Schuff N, Weiner M, et al. Patterns of brain atrophy in frontotemporal dementia and semantic dementia. Neurology. 2002;58:198-208.

Saxe R. Uniquely human social cognition. Curr Opin Neurobiol. 2006;16:235-9.

Schieber MH. Dissociating motor cortex from the motor. J Physiol. 2011;589:5613-24.

Serrien DJ, Kaluzny P, Wicki U, Wiesendanger M. Grip force adjustments induced by predictable load perturbations during a manipulative task. Exp Brain Res. 1999;124:100-6.

Shamay-Tsoory SG, Tomer R, Goldsher D, Berger BD, Aharon-Peretz J. Impairment in cognitive and affective empathy in patients with brain lesions: anatomical and cognitive correlates. J Clin Exp Neuropsychol. 2004;26:1113-27.

Shibuya K, Misawa S, Nasu S, Sekiguchi Y, Mitsuma S, Beppu M, et al. Split hand syndrome in amyotrophic lateral sclerosis: different excitability changes in the thenar and hypothenar motor axons. J Neurol Neurosurg Psychiatry. 2013;84:969-72.

Singer $\mathrm{T}$. The neuronal basis and ontogeny of empathy and mind reading: review of literature and implications for future research. Neurosci Biobehav Rev. 2006;30:855-63.

Snowden JS, Harris J, Richardson A, Rollinson S, Thompson JC, Neary D, et al. Frontotemporal dementia with amyotrophic lateral sclerosis: A clinical comparison of patients with and without repeat expansions in C9orf72. Amyotroph Lateral Scler Frontotemporal Degener. 2013;14:172-6. Stanton BR, Williams VC, Leigh PN, Williams SC, Blain CR, Giampietro VP, et al. Cortical activation during motor imagery is reduced in Amyotrophic Lateral Sclerosis. Brain Res. 2007;1172:145-51. Strong MJ. The syndromes of frontotemporal dysfunction in amyotrophic lateral sclerosis. Amyotroph Lateral Scler. 2008;9:323-38.

Stuss DT, Alexander MP. Executive functions and the frontal lobes: a conceptual view. Psychol Res. 2000;63:289-98.

Thomsen GM, Gowing G, Latter J, Chen M, Vit JP, Staggenborg K, et al. Delayed Disease Onset and Extended Survival in the S0D1G93A Rat Model of Amyotrophic Lateral Sclerosis after Suppression of Mutant SOD1 in the Motor Cortex. J Neurosci. 2014;34:15587-600.

Trimble M. Why humans like to cry - tragedy evolution, and the brain. Oxford: Oxford University Press; 2012.

Uddin LQ, Iacoboni M, Lange C, Keenan JP. The self and social cognition: the role of cortical midline structures and mirror neurons. Trends Cogn Sci. 2007;11:153-7.

Venkadesan M, Valero-Cuevas, F.J. Neural contol of motion-to-force transitions with the fingertip. J Neurosci. 2008;28:1366-73.

Vigneswaran G, Philipp R, Lemon RN, Kraskov A. M1 Corticospinal Mirror Neurons and Their Role in Movement Suppression during Action Observation. Curr Biol. 2013;23:236-43.

Virji-Babul N, Rose A, Moiseeva N, Makan N. Neural correlates of action understanding in infants: influence of motor experience. Brain Behav. 2012;2:237-42. 
Vucic S, Kiernan MC. Novel threshold tracking techniques suggest that cortical hyperexcitability is an early feature of motor neuron disease. Brain. 2006;129:2436-46.

Wang C, Wai Y, Kuo B, Yeh YY, Wang J. Cortical control of gait in healthy humans: an fMRI study. J Neural Transm. 2008;115:1149-58.

Weber M, Eisen A, Stewart H, Hirota N. The split hand in ALS has a cortical basis. J Neurol Sci. 2000;180:66-70.

Zaki J, Ochsner KN. The neuroscience of empathy: progress, pitfalls and promise. Nat Neurosci. 2012;15:675-80. 